\title{
ARTICLE OPEN \\ Socio-environmental drivers of sustainable adoption of household water treatment in developing countries
}

\author{
D. Daniel (iD), Sara J. Marks (iD ${ }^{2}$, Saket Pande ${ }^{1}$ and Luuk Rietveld ${ }^{1}$
}

Household water treatment (HWT) can effectively reduce exposure to unsafe drinking water at home. Understanding the characteristics of target groups who successfully adopt HWT, such as perception about water quality and usefulness of HWT, income, or parental education, is essential for enhancing the adoption of HWT in developing countries. The objective of this study is to analyze the interactions between such socio-environmental characteristics, rather than a single characteristic, in order to explain the adoption of HWT. Five socio-environmental characteristics and behavior determinants were analyzed using Qualitative Comparative Analysis (QCA) from 41 case studies in Africa, Asia, and South America. Results show that there is no single characteristic that alone explains the adoption of HWT. QCA identified five pathways leading to high adoption of HWT. Perceived threat due to bad water quality is a pre-condition for three of the pathways. However, perceived threat does not alone explain adoption of HWT and must be accompanied by other conditions. Households connected to piped water schemes can also be potential HWT adopters as long as they perceive poor tap water quality. Finally, households who are able to afford the full cost of HWT tend to adopt it only when they neither have prior experience with HWT nor a connection to a piped scheme. Our findings therefore highlight the necessity to analyze interactions between socio-environmental characteristics of households and behavior determinants in order to determine the adoption of HWT.

npj Clean Water (2018)1:12; doi:10.1038/s41545-018-0012-z

\section{INTRODUCTION}

Half of the world's population face severe water scarcity annually. ${ }^{1}$ This threatens the resilience of global water supplies and leads to high mortality and morbidity rate among children under the age of 5 years in developing countries, especially due to diarrheal diseases. ${ }^{2}$ Despite much progress in recent decades extending access to safely managed water services to $71 \%$ of global population, there are still 844 million people who do not have access to at least basic drinking water services. ${ }^{3}$ Moreover, about $40 \%$ of improved water sources are fecally contaminated or are at high risk of contamination. ${ }^{4}$

The 2030 United Nation Agenda for Sustainable Development explicitly focuses on water and sanitation management (Sustainable Development Goal (SDG) 6), with Target 6.1 aiming to achieve universal and equitable access to safe and affordable drinking water for all by $2030 .^{5}$ The long-term goal of SDG 6 is to provide safely managed drinking water meeting international standards for quality. ${ }^{6}$ However, financial, infrastructure, and human capital constraints are likely to limit the implementation of the SDG $6 .^{7-10}$

Household water treatment (HWT) technologies can safeguard public health in areas persistently challenged by efforts to achieve universal access to safe water. ${ }^{11}$ Several types of HWT technologies are being used, such as boiling, chlorination, and filtration, ${ }^{12,13}$ and newer technologies such as biochar and gravity-driven membrane-based HWT. ${ }^{14,15}$ HWT methods have been found to be more effective in improving household health than other types of interventions, such as treating water at the point of collection or at the source. ${ }^{16-18}$ Previous studies have shown that only those households that regularly treat their water experience the maximum health benefits of HWT methods (i.e., a sustained reduction in the rate of diarrhea). ${ }^{19}$ However, households often do not treat water regularly and even abandon HWT over time. ${ }^{20-22}$

Socio-environmental characteristics, like parental educational level or local culture, and behavior determinants, like perceived health threat due to bad water quality or willingness and ability to pay for a HWT product, have been found to influence successful adoption of HWT. ${ }^{23-25}$ Previous variable-driven experimental research and meta-analyses on HWT interventions have focused on testing statistical associations between individual socioenvironmental characteristics or behavior determinants and adoption of HWT. ${ }^{21,26,27}$ The question then is: do these characteristics and determinants alone influence the adoption of HWT? Or does an interaction between or combination of these characteristics and determinants best explain HWT adoption? If there is such combination, it has yet to be investigated, pointing to the necessity to understand how the characteristics of a socioenvironmental system may influence the adoption of HWT. ${ }^{11,28}$

Therefore, this paper aims to (1) determine whether a single or multiple interacting socio-environmental characteristics and behavioral determinants (called "conditions") best explain HWT adoption, and (2) if we cannot rely only on a single condition to explain adoption of HWT, then describe how conditions interact to influence the adoption of HWT. The results presented in this study draw on an extensive literature review of HWT adoption case studies in less developed countries.

\footnotetext{
${ }^{1}$ Water Management, TU Delft, Delft, the Netherlands and ${ }^{2}$ Sandec, EAWAG, Dübendorf, Switzerland
}

Correspondence: D Daniel (d.daniel@tudelft.nl)

Received: 25 October 2017 Revised: 16 March 2018 Accepted: 30 March 2018

Published online: 23 July 2018 


\section{RESULTS}

Necessity analysis

Necessity analysis assesses whether a factor is compulsory for the adoption of HWT. Table 1 shows the results of the necessity analysis for all conditions and their negation (negation indicated by a $\sim$ symbol in front of the condition name). The characteristics with the highest scores were $\sim$ EXHWT ( practice HWT, measured as $\leq 25 \%$ of households practiced HWT), followed by $\sim$ PIPE ( connected to pipe scheme, measured as $\leq 50 \%$ of households had access to a pipe scheme), and PERC (perceived threat, measured as $>50 \%$ of households perceived their water is bad and causes diseases). However, no condition had a consistency score above

Table 1. Consistency and coverage scores for each condition and its negation (indicated by $\sim$ ). Necessity analysis revealed that no individual condition was deemed necessary for high adoption rate of HWT

\begin{tabular}{|c|c|c|}
\hline Conditions & Consistency score & Coverage score \\
\hline Perceive threat (PERC) & 0.708 & 0.653 \\
\hline$\sim$ perceive threat $(\sim$ PERC) & 0.291 & 0.466 \\
\hline Practice HWT (EXHWT) & 0.208 & 0.333 \\
\hline$\sim$ practice HWT ( EXHWT) & 0.791 & 0.73 \\
\hline $\begin{array}{l}\text { Affordable to purchase HWT } \\
\text { product (AFFORD) }\end{array}$ & 0.708 & 0.586 \\
\hline $\begin{array}{l}\sim \text { affordable to purchase HWT } \\
\text { product }(\sim \text { AFFORD })\end{array}$ & 0.291 & 0.583 \\
\hline Connected to pipe scheme (PIPE) & 0.25 & 0.461 \\
\hline $\begin{array}{l}\sim \text { connected to pipe scheme } \\
\text { ( PIPE) }\end{array}$ & 0.75 & 0.642 \\
\hline $\begin{array}{l}\text { Parents completed primary } \\
\text { school (EDU) }\end{array}$ & 0.583 & 0.608 \\
\hline $\begin{array}{l}\sim \text { Parents completed primary } \\
\text { school }(\sim \text { EDU })\end{array}$ & 0.416 & 0.555 \\
\hline
\end{tabular}

0.9, indicating that no single condition was compulsory for successful implementation of HWT. Note that the consistency score measures how often a condition appears in the presence of the positive outcome. The higher the consistency score, the more often a condition appears in the presence of the positive outcome. Further, the condition AFFORD (affordable to purchase HWT product, measured as $>50 \%$ of households were able to afford the full cost of HWT) had the same consistency score as PERC (perceived threat) but had a slightly lower coverage value (Table 1). The coverage score, on the other hand, measures the proportion of positive case studies that are explained by a specific condition (in the case of necessity analysis) or the proportion of positive case studies which are represented by a specific pathway (in the case of sufficiency analysis).

\section{Sufficiency analysis}

The second step of QCA is sufficiency analysis, which identifies possible combinations of socio-environmental characteristics of target households for successful adoption of HWT. Sufficiency analysis provides one or more combinations of conditions (hereafter called pathways) that together are sufficient to lead to an outcome of interest. From the 41 case studies examined, 24 had high adoption rates and $83 \%$ of these (20 cases, from 15 countries, see Fig. 2) were explained by five pathways with a solution consistency score of 0.95 . All five pathways exceeded the consistency score threshold of 0.8 (Fig. 1), meaning that each were sufficient for explaining successful adoption of HWT.

The condition perceived threat (PERC) appeared in pathways 1,2 , and 3. Additionally, the condition PERC was also one of the top three conditions with the highest consistency score in the necessity analysis. Taken together, these findings suggest that PERC is an important condition to explain the adoption of HWT.

Pathway 1 represents low-income households with low education levels who did not depend on piped schemes for their main drinking water needs. Pathway 1 also reveals that high education of household members is not always necessary for successful adoption of HWT. A case from Pakistan (case study 4), for example, described that awareness programs, such as intensive water, sanitation, and hygiene (WASH) promotion activities delivered by

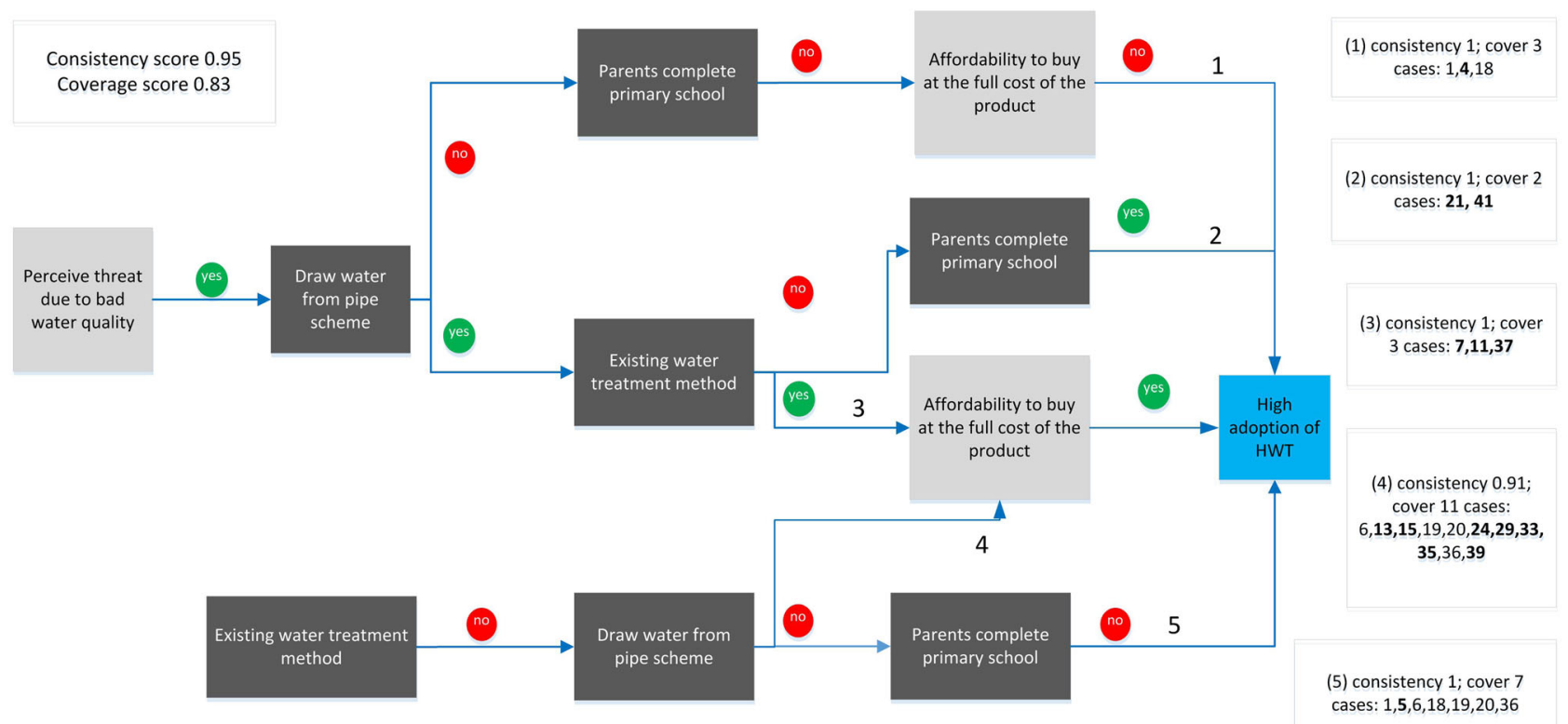

Fig. 1 Five causal pathways leading to high adoption of HWT. Bold numbers indicate unique coverage cases (i.e., cases that can only be explained by a specific pathway). "Yes" means "set-membership" and "no" means or "non-membership". Gray boxes represent behavior determinants and black boxes represent socio-environmental characteristics. See Fig. 2 for the country locations of the case studies 
NGOs, health care, or government, led to successful adoption of HWT in low-income poorly educated households. Additionally, the case study from Nepal (case study 1) emphasized that the target group highly appreciated the benefits of water filters, as demonstrated by a study participant who said "the filtered water appears clearer, tastes better, and smells better than the raw water". ${ }^{29}$

Pathways 2 and 3 represent households that also doubted the quality of water from their piped scheme. Cases in Pathway 2 suggest that higher education levels may lead to greater awareness of the health threats from poor water quality. Even though the households corresponding to Pathway 2 had a high awareness about water quality, the reason they did not treat their drinking water before the intervention is unclear. Moreover, the study in Malawi (case study 21) showed that continuous promotion by highly motivated health workers after the project finished, in combination with high levels of support from government, effectively achieved sustained HWT practices.

In contrast with Pathway 2, households in Pathway 3 had prior experience with treating their water. For example, in case 7 from Sri Lanka and case 37 from Guatemala, most households had already adopted the norm of boiling their drinking water before switching to ceramic filters that were distributed for free during an intervention. Since the pathway shows that households could afford more expensive products, apparently perceiving the benefits of a new HWT method played a role in their decision to replace their prior HWT method. Case 11 in Kenya revealed that appropriate promotion activities also played a role in successful adoption of HWT. In this case study, WaterGuard, a liquid chlorine solution, was promoted throughout the country and also integrated with an antenatal program.

Cases within Pathway 4 featured households that were dependent on non-piped sources and did not have prior experience with HWT but could afford a HWT product. Interestingly, for seven case studies in this pathway either perception of threat posed by poor water quality or high parental education level appeared as additional conditions that positively contributed to HWT adoption. Cases 6 (Madagascar), 19 (Cambodia), and 20 (Haiti) had low education levels but high perception of threat due to drinking untreated water. A high usage rate of chlorine solution was achieved because "almost all villagers were aware of the household disinfection strategy, and this knowledge was similar across literacy and socioeconomic strata". ${ }^{30}$ But cases 13 (Zambia), 15 (Guatemala), 33 (Bolivia), and 39 (Cambodia) showed the opposite, where households had a high education level but low perception of threat due to poor water quality.

Only one case can be categorized as being covered uniquely by pathway 5 (case 5 in the Democratic Republic of Congo). In case 5, households did not have a prior treatment method, did not have a piped water connection, and parents had not completed primary school. Nevertheless, the intervention led to a successful adoption. This exception may be explained by the free delivery of the product and a positive attitude of the people towards the product. The study mentions that the households liked the product because it improved the esthetic quality ( $88 \%$ of total intervention households) and taste (92\%) of water.

\section{DISCUSSION}

Our analysis revealed that no single condition could alone explain adoption of HWT. Instead, complex interactions among five socioenvironmental condition explained the adoption of HWT for 20 cases across 15 countries. These findings support the conclusion of a study by Clasen et al.,19 which states that "level of effectiveness may depend on a variety of conditions that research date cannot fully explain". Another important observation from this research is the interaction between socio-environmental characteristics and behavioral determinants (i.e., psychosocial factors), as seen in Pathways 1, 3, and 4 (see Fig. 1). In pathway 1 , for example, the type of water sources households used (socioenvironmental characteristics) appeared to influence their perception of their quality (psychosocial factor) and consequently influence their decision to use a HWT product. Several conceptual theories have attempted to link socio-environmental characteristics with psychosocial factors, e.g. IBM-WASH, ${ }^{25}$ health belief model $^{31}$ a model of communication for water treatment and safe storage, ${ }^{23}$ and RANAS. ${ }^{24}$ But analysis of such interactions remains a challenge.

Based on our analysis, households' perception that their own water quality is bad and risky to drink cannot alone explain the successful adoption of HWT. Yet these findings suggest that this condition is the most important precursor for successful adoption of HWT. Of 24 successful adoption cases, 17 cases (71\%) reported high perception of the risk of drinking untreated water. This finding aligns with several previous studies which concluded that negative perception of the quality of the water source is essential for successful adoption of HWT. ${ }^{32,33}$ This finding is also in line with a previous analysis from 10 countries, which concluded that negative perception of the quality of the water source caused households to purchase HWT products. ${ }^{34}$

The condition do not practice HWT ( EXHWT) also showed high consistency with the outcome of successful adoption of HWT. However, Pathway 3 featured cases where successful adoption was possible among households already practicing water treatment, representing an important target group for HWT interventions. Under such conditions, case details revealed that households perceiving the benefits of a new and affordable treatment method was an important driver for high adoption of new HWT. We therefore suggest that to maximize the likelihood of successful adoption in locations where water treatment is already being practiced, HWT implementers should target locations where existing treatment methods are not desirable and where households are willing and able to pay for a more effective product.

In summary, our comparative analysis reveals several insights for the implementation of HWT interventions. First, a system level approach that considers socio-environmental characteristics of households is needed when designing a HWT intervention program in less developed countries. Second, the absence of prior water treatment practices was the most consistent condition associated with successful adoption of HWT. We therefore recommend that interventions should target unserved regions where households do not have any prior experience with HWT, i.e., the focus should not be to introduce a new method of HWT to replace an existing HWT practices. Still, households already practicing treatment may decide to adopt a new HWT method if it is affordable and confers tangible benefits over the existing method; thus, implementers should also focus on this target group. Third, perception of water being risky to drink is a consistent precursor to successful adoption of HWT. Thus we recommend that assessing the perception of households should be the focus of any pre-intervention program. If households do not perceive water quality as bad, education and awareness programs should be initiated before the introduction of HWT. Lastly, two pathways showed that people who draw water from piped schemes could adopt HWT provided that the households perceive that water quality is bad. This suggests that HWT is not a competitor for piped schemes, but instead serves to complement it. Piped water suppliers should include HWT implementation if they cannot guarantee good water quality at the point of collection.

\section{METHODS}

Qualitative comparative analysis

Qualitative comparative analysis (QCA) was used to comparatively analyze 41 case studies to identify combinations of conditions (pathways) leading 
to successful adoption of HWT. In QCA all explanatory variables are called conditions. QCA provides necessity analysis to identify necessary conditions (i.e., a condition that must appear) to generate an outcome of interest, and sufficiency analysis to identify one or more possible pathways for achieving the outcome of interest. The "goodness of fit" of necessary and sufficient conditions is assessed in terms of "consistency" and "coverage" scores. The consistency score measures the degree to which a condition explains a positive outcome, and the coverage score measures the proportion of case studies that are explained by a specific pathway. Consistency and coverage score thresholds of 0.9 and 0.3 , respectively, were used to determine necessary conditions. A consistency score threshold of 0.8 was used to determine sufficient conditions. ${ }^{35-37}$

We used crisp set Qualitative Comparative Analysis (csQCA), which makes use of binary input data. Conditions describe socio-environmental characteristics and are based on bivalent logic with prescribed thresholds, i.e., either present/true (1) or absent/false (0). CsQCA is the simplest method in QCA and may oversimplify the system, but is still capable of providing useful insight. This study made use of fSQCA 2.5 software (compasss.org). All data were encoded in Excel and saved in.csv format as input to the software. The intermediate solution without prior assumption was used to perform the analysis.

\section{Case selection}

An extensive review of peer-reviewed literature on HWT interventions was conducted. The review was limited to articles written in English. We did not distinguish between real-world implementations, intervention trials, or interventions after an emergency situations, such as HWT interventions after flooding events. No restrictions such as location, type of HWT, year published, or year conducted were put on the selection of cases. The main inclusion criterion for case study selection was the assessment time, defined as the duration of time between introduction of HWT and measurement of its usage. All papers that described case studies with an assessment time greater than 12 months were eligible for inclusion.

In total, 41 case studies met the inclusion criteria. These were published during 2003-2016, offered five main types of HWT technologies (chlorination, flocculation, filtration, UV light, and pasteurization) across 24 countries and 4 continents (Fig. 2).

\section{Identifying causal conditions and the outcome}

Five socio-environmental characteristics and behavior determinants (or conditions) were determined from the review and examined in this study: affordability to purchase a HWT product at full cost (AFFORD), perceived threat due to bad water quality (PERC), type of domestic water source (PIPE), existing HWT before the intervention or proportion of households who already used HWT (EXHWT), and parental education level (EDU). PERC and AFFORD represent behavior determinants, while PIPE and EDU represent socio-environmental characteristics of the community. EXHWT can represent both behavior determinant (descriptive norms according to RANAS $^{24}$ ) and socio-environmental characteristics. These two elements (i.e., socio-environmental characteristics and behavior determinants) were analyzed separately and considered to be of no distinct relation to each other. $^{25}$

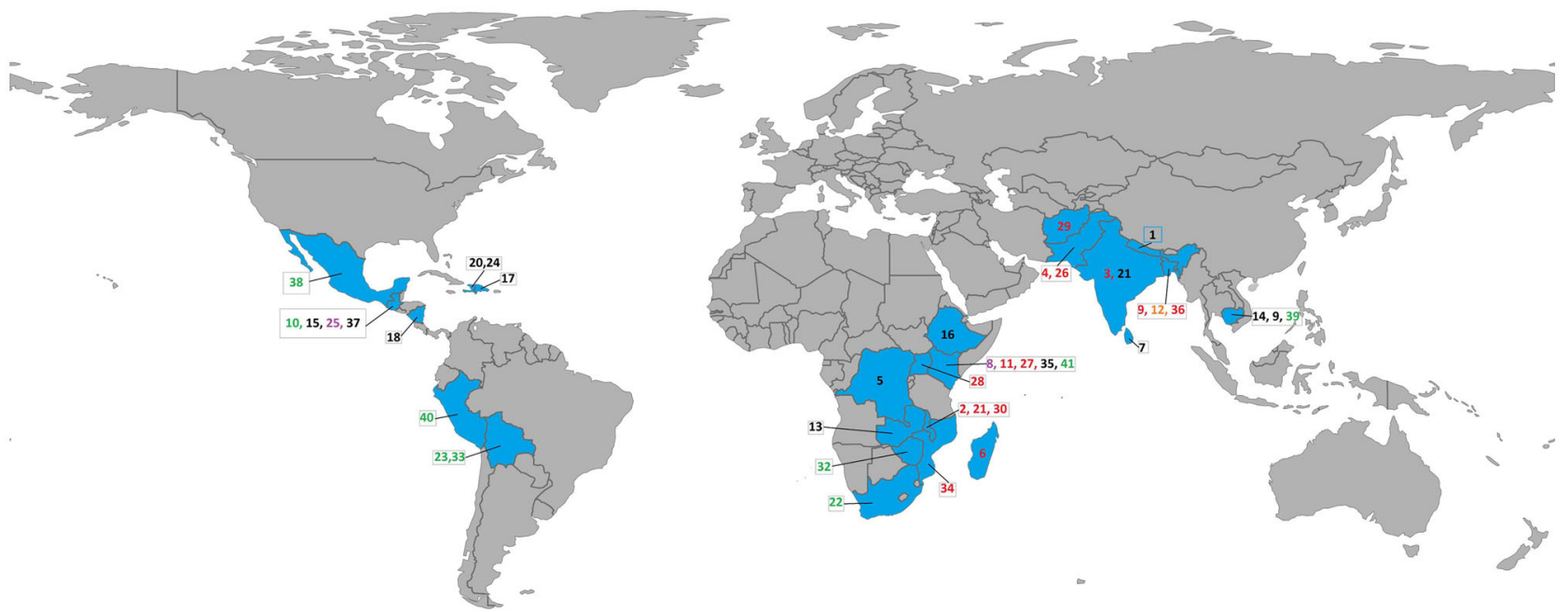

Fig. 2 Locations of the case studies selected for QCA (i.e., including both successful and unsuccessful cases). The numbers indicate the case numbers. The color of the text indicates the type of HWT intervention. Black $=$ filtration, red $=$ chlorination, purple $=$ flocculation, green $=$ SODIS (UV light), orange = pasteurization

Table 2. Coding rubric developed to score outcome and causal conditions

Variables (causal conditions and outcome)

Outcome: Adoption rate (ADOPT)

Perceive thread (PERC)

Existing HWT before intervention (EXHWT)

Affordability (AFFORD)

Connection to pipe scheme (PIPE)

Parental education level (EDU)
Coding scheme and threshold

1: Adoption rate of HWT $>50 \%$

0 : Adoption rate of HWT $\leq 50 \%$

1: $>50 \%$ of households perceived their water is bad and causes diseases

0 : $\leq 50 \%$ of households perceived their water is bad and causes diseases

1: $>25 \%$ of households practicing any kind of household water treatment

0 : $\leq 25 \%$ of households practicing any kind of household water treatment

1: $>50 \%$ of households in the study area being able to afford the full cost of HWT products $0: \leq 50$ of households in the study area being able to afford full cost of the HWT products

1: $>50 \%$ of households in the study area draw water from a piped scheme

$0: \leq 50$ of households in the study area draw water NOT from pipe scheme

1: $>50 \%$ parents in the study area had completed primary school

$0: \leq 50 \%$ parents in the study area had completed primary school 
The main criteria for the inclusion of conditions in the analysis were conformity with the literature, consistent availability of data across the case studies examined and added value to the analysis. The adoption rate of HWT was defined as the outcome variable (ADOPT). Since no standard definition of high adoption of HWT existed at the time of the study, we set $50 \%$ adoption rate as the threshold for high adoption of HWT. Table 2 summarizes the threshold for each causal condition and outcome variable. Each study case was assigned full membership (coding 1) or full nonmembership (coding 0) and the membership score are reported in supporting information (Table S2).

We did not include other factors that may influence adoption rate in this analysis, such as subsidies, intensive promotion activities, durability of the product, or household's preference for a specific type of HWT item (see S1 for more information about the selection of causal conditions). Most of the data were obtained from the literature, with missing relevant information obtained by querying the article's author or gathering information from other relevant studies in the same area.

Data availability

The authors declare that the data supporting the findings of this study are available within the paper and the supplementary materials.

\section{ACKNOWLEDGEMENTS}

D.D. is a PhD candidate at TU Delft and receives funding from Indonesia Endowment Fund for Education (LPDP). The funders played no role in the study design, the development of this manuscript, or the decision to publish. We also thank Arifin Chun for creating the map of case studies and Johanna Lie for writing advice.

\section{AUTHOR CONTRIBUTIONS}

Conceived and designed the research: S.P. Conducted the research: D.S. Analyzed the results: D.S., S.P., S.M., and L.R. Wrote the first draft of the manuscript: D.S. Contributing to the writing of the article: D.S., S.P., S.M., and L.R.

\section{ADDITIONAL INFORMATION}

Supplementary information accompanies the paper on the npj Clean Water website (https://doi.org/10.1038/s41545-018-0012-z).

Competing interests: The authors declare no competing interests.

Publisher's note Springer Nature remains neutral with regard to jurisdictional claims in published maps and institutional affiliations.

\section{REFERENCES}

1. Mekonnen, M. M., \& Hoekstra, A. Y. Four billion people facing severe water scarcity. Sci. Adv. 2, e1500323 (2016)

2. Colombara, D. V. et al. Chronic health consequences of acute enteric infections in the developed world. Am. J. Gastroenterol. 3, 12-24 (2016).

3. WHO; UNICEF. Progress on Drinking Water, Sanitation and Hygiene: 2017 Update and SDG baselines. Geneva: WHO and UNICEF (2017).

4. Onda, K., LoBuglio, J. \& Bartram, J. Global access to safe water: accounting for water quality and the resulting impact on MDG progress. Int. J. Environ. Res. Public Health 9, 880-894 (2012).

5. UNWater. The United Nations World WaterDevelopment Report. Water for a Sustainable World. Paris: UNESCO (2015).

6. World Health Organization. Guidelines for Drinking-Water Quality 4th ed. (World Health Organization, 2011). https://doi.org/10.1016/S1462-0758(00)00006-6.

7. Jagals, P. Does improved access to water supply by rural households enhance the concept of safe water at the point of use? A case study from deep rural South Africa. Water Sci. Technol. 54, 9 (2006).

8. Hutton, G. \& Bartram, J. Global costs of attaining the Millennium Development Goal for water supply and sanitation. Bull. World Health Organ. 86, 13-19 (2008).

9. WHO. Combating Waterborne Disease at the Household Level/International Network to Promote Household Water Treatment and Safe Storage. Geneva: World Health Organization (2007).

10. Johnston, R. B., Hanchett, S. \& Khan, M. H. The socio-economics of arsenic removal. Nat. Geosci. 3, 2-3 (2010).

11. Clasen, T. Scaling up Household Water Treatment among Low-income Populations (World Health Organization, Geneva, 2009).
12. Lantagne, D. S., Quick, R. E. \& Mintz, E. D. Household water treatment and safe storage options in developing countries: a review of current implementation practices. Woodrow Wilson: International Center for Scholars' Environmental Change and Security Program (2011).

13. WHO Western Pacific Region. Household Household Water Water Treatment Treatment and Safe Storage Manual for Participant. Manila: World Health Organization (2013).

14. Gwenzi, W., Chaukura, N., Noubactep, C. \& Mukome, F. N. D. Biochar-based water treatment systems as a potential low-cost and sustainable technology for clean water provision. J. Environ. Manag. 197, 732-749 (2017).

15. Peter-Varbanets, M. et al. Gravity driven membrane disinfection for household drinking water treatment. In 35th WEDC International Conference, Loughborough, UK (pp. 1-8). Loughborough: WEDC International Conference (2011).

16. Sobsey, M. D., Stauber, C. E., Casanova, L. M., Brown, J. M. \& Elliott, M. A. Point of use household drinking water filtration: a practical, effective solution for providing sustained access to safe drinking water in the developing world. Environ. Sci. Technol. 42, 4261-4267 (2008).

17. Clasen, T. F. \& Mintz, E. D. International network to promote household water treatment and safe storage. Emerg. Infect. Dis. 10, 1179 (2004).

18. Padilla, D. The Effectiveness of Water, Hygiene, and Sanitation Interventions in Lowering Diarrheal Morbidity Across The Globe: A Systematic Review and Qualitative Analysis of Relevant Primary Literature. Arizona: Arizona State University (2012).

19. Clasen, T., Schmidt, W.-P., Rabie, T., Roberts, I. \& Cairncross, S. Interventions to improve water quality for preventing diarrhoea: systematic review and metaanalysis. BMJ 334, 782 (2007).

20. Waddington, H. \& Snilstveit, B. Effectiveness and sustainability of water, sanitation, and hygiene interventions in combating diarrhoea. J. Dev. Eff. 1, 295-335 (2009).

21. Hunter, P. R. Household water treatment in developing countries: comparing different intervention types using meta-regression. Environ. Sci. Technol. 43, 8991-8997 (2009).

22. Schmidt, W. \& Cairncross, S. Household water treatment in poor populations: is there enough evidence for scaling up now? Household water treatment in poor populations: is there enough evidence for scaling up now? Environ. Sci. Technol. 43, 986-992 (2009)

23. Figueroa, M. \& Kincaid, D. Social, cultural and behavioral correlates of household water treatment and storage. Cent. Publ. HCl2010-1 Heal. Commun. Insights, Balt. Johns Hopkins Bloom. Sch. Public Heal. Cent. Commun. Programs 0-55 (2010).

24. Mosler, H. J. A systematic approach to behavior change interventions for the water and sanitation sector in developing countries: a conceptual model, a review, and a guideline. Int. J. Environ. Health Res. 22, 431-449 (2012).

25. Dreibelbis, R. et al. The integrated behavioural model for water, sanitation, and hygiene: a systematic review of behavioural models and a framework for designing and evaluating behaviour change interventions in infrastructurerestricted settings. BMC Public Health 13, 1015 (2013).

26. Inauen, J., Hossain, M. M., Johnston, R. B. \& Mosler, H. J. Acceptance and use of eight arsenic-safe drinking water options in Bangladesh. PLOS ONE 8, e53640 (2013).

27. Loharikar, A. et al. Long-term impact of integration of household water treatment and hygiene promotion with antenatal services on maternal water treatment and hygiene practices in Malawi. Am. J. Trop. Med. Hyg. 88, 267-274 (2013).

28. Cairncross, S. Sanitation and Water Supply: Practical Lessons from the Decade. World Bank (1992)

29. Ngai, T. K. K., Shrestha, R. R., Dangol, B., Maharjan, M. \& Murcott, S. E. Design for sustainable development-household drinking water filter for arsenic and pathogen treatment in Nepal. J. Environ. Sci. Heal. A 42, 1879-1888 (2007).

30. Ram, P. K. et al. Bringing safe water to remote populations: an evaluation of a portable point-of-use intervention in rural Madagascar. Am. J. Public Health $\mathbf{9 7}$ 398-400 (2007).

31. Rainey, R. C. \& Harding, A. K. Acceptability of solar disinfection of drinking water treatment in Kathmandu Valley, Nepal. Int J. Env. Heal. Res 15, 361-372 (2005).

32. Nagata, J. M. et al. Criticisms of chlorination: social determinants of drinking water beliefs and practices among the Tz'utujil Maya. Rev. Panam. Salud Publica 29, 9-16 (2011).

33. Harris, J. Challenges to the commercial viability of point-of-use (POU) water treatment systems in low-income settings. Am. J. Med. Genet. A 155, fmi-v (2005).

34. Johnstone, N. \& Serret, Y. Determinants of bottled and purified water consumption: results based on an OECD survey. Water Policy 14, 668-679 (2012).

35. Sehring, J., Korhonen-Kurki, K. \& Brockhaus, M. Qualitative comparative analysis (QCA) an application to compare national REDD+policy processes. Cent. Int. For. Res. 34 (2013). https://doi.org/10.17528/cifor/004278

36. Ragin, C. C. Redesigning social inquiry. Econ. Soc. Res. Counc. Res. Methods Festival, Saint Catherine's Coll. Oxford Univ. 1-30 (2008). https://doi.org/10.7208/chicago/ 9780226702797.001 .0001 
37. Legewie, N. An introduction to applied data analysis with qualitative comparative analysis. Forum Qual. Sozialforschung/Forum Qual. Soc. Res. (2013). http://www. qualitative-research.net/index.php/fqs/article/view/1961/3594 (accessed 24 January 2017)

(c) Open Access This article is licensed under a Creative Commons Attribution 4.0 International License, which permits use, sharing, adaptation, distribution and reproduction in any medium or format, as long as you give appropriate credit to the original author(s) and the source, provide a link to the Creative
Commons license, and indicate if changes were made. The images or other third party material in this article are included in the article's Creative Commons license, unless indicated otherwise in a credit line to the material. If material is not included in the article's Creative Commons license and your intended use is not permitted by statutory regulation or exceeds the permitted use, you will need to obtain permission directly from the copyright holder. To view a copy of this license, visit http://creativecommons. org/licenses/by/4.0/.

(c) The Author(s) 2018 